\title{
ANÁLISIS DE LAS APLICACIONES MÓVILES DE LAS TELEVISIONES GENERALISTAS ESPAÑOLAS Y PORTUGUESAS: INTERACTIVIDAD Y CONTENIDOS
}

\section{Analysis of the Mobile Applications of the Generalist Spanish and Portuguese Televisions: Interactivity and Content}

\author{
Dr. José Juan VIDELA RODRÍGUEZ \\ Profesor Contratado Doctor, Universidade da Coruña, España \\ E-mail: videla@udc.es
}

(iD https://orcid.org/0000-0001-8656-9297

\section{Dra. María Josefa FORMOSO BARRO}

Profesor Ayudante Doctor, Universidade da Coruña, España

E-mail: m.formoso@udc.es

(iDhttps://orcid.org/0000-0002-9561-4915

\author{
Dr. Manuel GARCÍA TORRE \\ Contratado Interino de Sustitución, Universidade da Coruña, España \\ E-mail: manuel.garcia.torre@udc.es \\ (D) https://orcid.org/0000-0002-9016-1267
}

Fecha de recepción del artículo: 01/10/2018

Fecha de aceptación definitiva: 23/10/2018

\begin{abstract}
RESUMEN
Este artículo analiza los contenidos y opciones interactivas ofrecidas en las aplicaciones móviles para tablets con sistemas operativos iOS y Android por parte de las televisiones generalistas españolas y portuguesas. Para ello se realiza un análisis a través de una ficha de elaboración propia, de las televisiones objeto de estudio: RTVE, ATRESMEDIA, MEDIASET, RTP, TVI y SIC. Se destaca que las televisiones españolas ofrecen una mayor oferta de contenidos y mayores opciones de interacción entre usuario y medio.

Palabras clave: aplicaciones móviles; contenidos audiovisuales; televisión; interactividad; tabletas.

ABSTRACT

This article makes an approach to the content and interactive options offered in mobile apps for tablets with Android and iOS operating systems by the Generalist Spanish and Portuguese television. This is an analysis through an ad hoc model that includes the different television channels included in the study: RTVE, ATRESMEDIA, MEDIASET, RTP, TVI and SIC. This analysis is highlighted the fact that Spanish television channels offer a wider range of content and more options of interaction between users and the media.

Key words: mobile applications; audiovisual content; television; interactivity; tablet.
\end{abstract}




\section{INTRODUCCIÓN}

La comunicación móvil se ha convertido en el paradigma de una nueva forma de acceder a los contenidos audiovisuales de información y entretenimiento. La telefonía móvil e Internet caracterizan la era digital en la que estamos inmersos, provocando cambios que afectan al desarrollo social, económico y cultural de nuestras sociedades (Igarza, 2009). Nuestra actividad diaria ya no se concibe sin el recurso del dispositivo móvil, bien sea para una acción profesional, bien para las relaciones personales o el ocio, a tal punto que probablemente sea el dispositivo tecnológico más importante del ser humano en este tiempo (Márquez, 2015).

Desde que la pantalla del ordenador acabó con el monopolio de la televisión como fuente de entretenimiento audiovisual doméstico se han ido sucediendo una serie de innovaciones tecnológicas que han afectado a toda la cadena de negocio y han provocado profundas transformaciones en la forma en que las empresas audiovisuales explotan sus contenidos, tanto en la distribución como en la recepción, rompiendo las rígidas estructuras anteriores a la revolución digital (Vila Fumas \& Fuentes Cortina, 2013).

Por lo que respecta al consumo hay que considerar la realización de tareas simultáneas, no solo en aparatos distintos, multi-device, sino también el acceso simultáneo a varias fuentes de contenido en un mismo dispositivo, multi-window (Fernández-Planells \& Figueras Maz, 95). En este sentido, y en lo que atañe específicamente a las tablets, el 58\% de los encuestados para el informe \#IAB Estudio Mobile indicaron que utilizan este dispositivo como segunda pantalla mientras ven la televisión, con los juegos, redes sociales y móviles como actividades principales; también el estudio TV or not TV, that's the question de Zenith (2015) refleja que en el nuevo ecosistema de medios el 49\% de los espectadores utilizan otro dispositivo mientras ven la televisión. Estas se han convertido en grandes contenedores que presentan su oferta en distintas ventanas de recepción. Este proceso no ha avanzado en la experimentación de nuevos formatos específicos para movilidad ya que las emisoras recurren fundamentalmente a los programas y formatos de la emisión convencional para nutrir su oferta en movilidad, como señalan Pérez Rufí y Gómez Pérez (2013) y F. Formoso (2015).

Nuestra investigación se ha centrado en la distribución de contenidos audiovisuales televisivos a través de las tabletas, un dispositivo mucho menos extendido que los teléfonos inteligentes.

\section{MARCO TEÓRICO}

El crecimiento espectacular de la penetración de los dispositivos móviles los ha convertido, especialmente en el caso de los teléfonos, en «el primer medio de comunicación interpersonal» (Fidalgo, 2009, 123), y ha trascendido su funcionalidad como vehículo de transmisión para generar nuevos formatos, sistemas de financiación y hasta un lenguaje propio (Vacas, 2007).

La denominada cuarta pantalla (Cebrián \& Flores Vivar, 2011; Aguado \& Martínez, 2008) es un instrumento que ha potenciado la interacción y la participación de la audiencia; esta se ha convertido también en un objeto de investigación relevante (Loosen \& Schmidt, 2012) desde una triple consideración: como receptores, productores o redes (Nel \& Westlund, 2013).

Las investigaciones académicas sobre la comunicación móvil han ido cobrando relevancia en número y profundidad a medida que ha ganado valor uso desde el punto de vista de los emisores y de los receptores, de tal manera que se ha avanzado en varias direcciones de análisis: la oferta y estructura de contenidos, los modelos de negocio y los usos por parte de los receptores (Costa-Sánchez, Díaz González \& Videla-Rodríguez, 2012). 
Por lo que respecta a las investigaciones y proyectos en las universidades, en España se desarrollan varias líneas que parten del concepto global de comunicación móvil para buscar su desarrollo en diferentes dispositivos. En Galicia, el Grupo de Cultura y Comunicación Interactiva de la Universidade da Coruña ha partido del concepto global de interactividad para aplicarlo específicamente a la comunicación en movilidad, aunque este no ha sido este el único objeto de investigación en este campo, ya que los trabajos de investigación han abordado los contenidos en movilidad audiovisuales y periodísticos. En este sentido cabe destacar los trabajos sobre la plataforma smartphone (Costa-Sánchez, 2014 y 2013), entorno iOS (Sanjuán et al., 2013), las aplicaciones de radio para smartphones (VidelaRodríguez \& Piñeiro-Otero, 2013; Piñeiro-Otero, 2015a; 2015b), las de televisión (García-Torre, 2014) y también en torno a los contenidos informativos en movilidad de los medios públicos españoles (Videla Rodríguez, García-Torre \& Formoso Barro, 2014).

En la Universidad de Santiago desarrolla su actividad el grupo de investigación en Novos Medios, que desde el ámbito del Periodismo se ha ocupado del desarrollo de los cibermedios en España y América, también de la arquitectura de la información con atención específica a los dispositivos móviles. Dentro de las investigaciones de los miembros de este grupo destacan los trabajos del profesor López García sobre la incidencia de los dispositivos móviles en las industrias culturales (CostaSánchez, Rodríguez Vázquez \& López García, 2016) y los contenidos, comercio y conversación a través de la comunicación móvil (Silva Rodríguez, López García \& Westlund y Ulloa Erazo, 2016).

La Universidad de Murcia cuenta con el grupo eCOM, que ha trabajado en proyectos sobre las implicaciones de la telefonía móvil en la sociedad española desde un punto de vista cultural y tecnológico social (Aguado \& Martínez, 2006 y 2008). El Grupo de Investigación de Interacciones Digitales de la Universidad de Vic la telefonía móvil desde varios puntos de vista. Cobo Romaní y Pardo Kuklinski (2007) han jugado un papel fundamental en la difusión de esta línea de investigación con la publicación de Planeta Web 2.0: Inteligencia colectiva o medios fast food.

En la Universidad de Navarra desarrolla su actividad el Center for Internet Studies and Digital Life que ha investigado sobre el impacto de las transformaciones digitales en la comunicación, con diversa orientación: redes sociales, periodismo digital, nuevos modelos de negocio o publicidad interactiva. La prospectiva sobre las transformaciones que se pueden producir a causa de las nuevas formas de comunicación queda reflejada en los trabajos de Salaverría (2016) y Pérez-Latre y SánchezTabernero (2016).

En el ámbito europeo destacan los trabajos de Oscar Westlund sobre el nuevo paradigma de la comunicación móvil y sus implicaciones para el periodismo. Su tesis doctoral Cross-media News Work Sensemaking of the Mobile Media (R)evolution (2011) es un estudio de referencia en este campo de investigación; su foco de atención también se ha centrado, entre otros aspectos, en los patrones de consumo (2015).

El Labcom de la Universidade da Beira Interior, en Portugal, es relevante en las investigaciones sobre comunicación móvil. Los trabajos liderados por el profesor Joao Canavilhas han analizado el producto con especial atención a las plataformas del entorno iOS (Canavilhas, 2009; Fidalgo \& Canavilhas, 2009; Canavilhas \& Santana, 2011; Canavilhas, 2012).

En el norte de Europa cabe destacar que el IVTT Technical Research Centre of Finland es pionero en el estudio de plataformas móviles como instrumento de comunicación. En Suecia se encuentra el Mobile Life Research Centre, especializado desde 2007 en el estudio de la interactividad, las aplicaciones y los nuevos dispositivos móviles. 
Fuera del continente europeo sobresalen las investigaciones que en la University of Sidney coordina el profesor Gerard Goggin, pionero en los estudios sobre comunicación móvil y los medios. También es el editor de Studies in Mobile Communication series (Oxford University Press con Rich Ling), y forma parte del equipo editorial de Mobile Media and Communication (Sage).

Desde Estados Unidos el Centro de Estudios de la Comunicación Móvil en la Escuela de la Universidad de Rutgers, fundada en 2004 y dirigida por James E. Katz, investiga las consecuencias de la revolución social, psicológica y organizacional de las comunicaciones móviles.

En Brasil, se encuentra en marcha el Projeto Laboratório de Jornalismo Convergente, de la Facultad de Comunicación de la Universidade Federal da Bahía (Brasil). Allí se han realizado investigaciones sobre el nuevo paradigma periodístico en movilidad (Barbosa \& Seixas, 2013).

Las aplicaciones como creación y mediador tecnológico para la distribución de contenidos en movilidad también están despertando un interés creciente por parte de los investigadores. Si bien el acercamiento se está produciendo fundamentalmente para analizar su incidencia sobre el negocio y la difusión, se constatan casos de estudio que analizan aplicaciones por sectores de negocio (Ford \& Wiedemann, 2011), el diseño de las apps (Cuello \& Vittone, 2013) o las diferencias entre la web y las aplicaciones móviles (Brognara, 2016).

No solo podemos reflejar estudios e investigación básica y aplicada en el ámbito de la academia. Diversas empresas de comunicación en todo el mundo han desarrollado unidades de investigación e innovación bajo la forma de medialabs o laboratorios de medios para asumir e implementar las transformaciones digitales que se están produciendo en la comunicación social. Se han centrado más en la experimentación de contenidos multimedia y proyectos de visualización más que en desarrollo de herramientas o aplicaciones tecnológicas (Salaverría \& Sádaba, 2016). En España han tenido escasa incidencia ya que hasta la fecha solo los han desarrollado el Diario de Navarra, El Confidencial, RTVE y el Grupo Vocento; también El Mundo y La Vanguardia han creado unidades para la innovación periodística en el ámbito del multimedia y el periodismo de datos.

\section{3. ОвJETIVOS}

Este trabajo muestra la evolución de los contenidos en las aplicaciones móviles de los operadores generalistas de televisión en España y Portugal. Efectúa un análisis comparativo de la oferta de contenidos de entretenimiento y las herramientas interactivas en tabletas.

Los objetivos de este estudio consisten en encuadrar los contenidos que los operadores españoles de televisión (RTVE, Atresmedia y Mediaset) y los operadores portugueses (RTP, TVI y SIC) distribuyen en las aplicaciones para dispositivos móviles y, en concreto, en las tabletas.

\section{Se establecen dos objetivos principales:}

En primer lugar, comprobar si los contenidos se adecuan al dispositivo móvil o se limitan a difundir material emitido en los canales de televisión de cada uno de los operadores.

En segundo lugar, constatar si las posibilidades interactivas que caracterizan a estos dispositivos son utilizadas y, así, confirmar si el espectador es un mero receptor o participa activamente en la creación de los contenidos.

Como objetivos secundarios se examinan los recursos que se manejan en las tabletas y se realiza una comparativa con la finalidad de establecer si hay diferencia entre dispositivos iOS y Android en cada uno de los canales de televisión de los operadores mencionados portugueses y españoles. 
También se determina la evolución del grado de innovación y desarrollo de los contenidos en movilidad de los grupos audiovisuales analizados.

\section{Metodología}

Para llegar a los objetivos planteados, se desarrolla un análisis de las aplicaciones móviles de contenidos generales y las interactivas que pertenecen a los grupos de comunicación televisivos públicos y privados de ámbito estatal en España y Portugal. El objeto concreto de estudio son las apps de estas televisiones que están disponibles en tabletas con sistemas operativos iOS y Android. A continuación se presentan (tabla 1) las aplicaciones móviles analizadas:

Tabla 1: Aplicaciones móviles analizadas

\begin{tabular}{|c|c|c|}
\hline $\begin{array}{c}\text { Televisión pública o privada de } \\
\text { ámbito estatal }\end{array}$ & Apps para tableta iOs & Apps para tableta Android \\
\hline RTVE & $\begin{array}{c}\text { Rtve.es | Tableta } \\
+ \text { tve }\end{array}$ & $\begin{array}{c}\text { Rtve.es | Tableta } \\
+ \text { tve }\end{array}$ \\
\hline Atresmedia & $\begin{array}{c}\text { Atresplayer } \\
\text { Atresmedia conecta }\end{array}$ & $\begin{array}{c}\text { Atresplayer } \\
\text { Atresmedia conecta }\end{array}$ \\
\hline Mediaset & Mitele & $\begin{array}{c}\text { Mitele } \\
\text { Mio TV }\end{array}$ \\
\hline RTP & $\begin{array}{c}\text { RTP } \\
\text { RTP Play }\end{array}$ & RTP \\
\hline RVI & TVI & TVI \\
\hline SIC & TVI Player & TVI Player \\
\hline
\end{tabular}

Fuente: elaboración propia

A esta muestra seleccionada, se le aplica, a través de técnicas cuantitativas, una ficha de análisis que cuenta con dos apartados principales que estudian los contenidos y las herramientas interactivas disponibles. Dentro de estos apartados hay diferentes ítems, como, por ejemplo, en lo que se refiere a los contenidos, algunos hacen referencia a cuál es la estructura de los contenidos de entretenimiento, si hay publicidad, si se ofrece la posibilidad de acceder a contenidos extra o si hay oferta de emisiones en directo. En el caso de la interactividad, se revisan las opciones a la hora de compartir un contenido, si se ofrece la posibilidad de interactuar en directo o la participación de los usuarios a partir de comentarios, votaciones, etc. Además del contenido y de la interactividad, también se analizan otros aspectos como son el tipo de app que nos encontramos (corporativa o de un canal concreto), así como su finalidad, contenidos y/o interactividad. El análisis de las aplicaciones se ha realizado entre los meses de marzo y mayo del año 2018. A continuación, se puede ver la ficha (tabla 2) utilizada: 
JOSÉ JUAN VIDELA RODRÍGUEZ, MARÍA JOSEFA FORMOSO BARRO Y MANUEL GARCÍA TORRE ANÁLISIS DE LAS APLICACIONES MÓVILES DE LAS TELEVISIONES GENERALISTAS ESPAÑOLAS Y...

Tabla 2: Ficha de análisis del contenido e interactividad

\begin{tabular}{|c|c|}
\hline \multirow{2}{*}{ TIPO DE $A P P$} & Corporativa \\
\hline & Canal \\
\hline \multirow{2}{*}{ FINALIDAD $A P P$} & Contenidos \\
\hline & Interactividad \\
\hline \multirow{22}{*}{ CONTENIDO } & Secciones de la oferta general de la aplicación. \\
\hline & Estructura entretenimiento \\
\hline & Categorías destacadas \\
\hline & Más vistos \\
\hline & Orden alfabético \\
\hline & Recomendados \\
\hline & Publicidad \\
\hline & Vídeo \\
\hline & Banner. Formatos expandibles, integrados, flotantes \\
\hline & Ventana flotante \\
\hline & Videoteca (contenidos anteriores. Limitada/extensa) \\
\hline & Capítulos \\
\hline & Programas \\
\hline & Contenidos extra \\
\hline & Información complementaria \\
\hline & Fragmentos \\
\hline & Extras \\
\hline & Segunda pantalla \\
\hline & Encuestas \\
\hline & Producción propia \\
\hline & Producción ajena \\
\hline & Directo \\
\hline \multirow{5}{*}{ INTERACTIVIDAD } & Conexión a redes sociales \\
\hline & Contenidos generados por usuarios \\
\hline & Compartir contenidos \\
\hline & Interacción en directo \\
\hline & $\begin{array}{l}\text { Participación de los usuarios (votaciones, comentarios, comunidades de } \\
\text { seguidores...) }\end{array}$ \\
\hline
\end{tabular}

Fuente: elaboración propia 


\section{RTVE}

RTVE tiene dos aplicaciones para iPad y tableta Android: Rtve.es | Tablet, estructurada por secciones de oferta: «Noticias», «Deportes», «TV a la carta», «Radio a la carta», «TVE en Directo», «RNE en Directo»; +tve, aplicación orientada a la interacción y participación del usuario, aunque en la aplicación general también se prevén esas opciones, y 24 Horas RTVE dedicada en exclusiva a contenidos informativos.

Rtve.es | Tableta, es una aplicación corporativa que tiene una oferta general estructurada en los siguientes apartados: «Noticias», «Deportes», «Somos Documentales», «TV a la carta», «Radio a la carta», «TVE en Directo» y «RNE en Directo»; también hay un acceso específico a RNE.

El entretenimiento está basado en tres categorías: «Series», «Documentales» y «Programas» a la que se accede a través de TVE a la carta. En cada una de ellas se segmenta el contenido en: «Todos los programas», «En Emisión», «Fuera de emisión»y «Orden Alfabético». La oferta de videoteca es amplia, con capítulos y programas.

Los contenidos extra ofrecen información complementaria, referida a la trama, argumento o contenido del programa, así como fragmentos de la producción, que siempre es de elaboración propia. Hay contenido en directo.

Por lo que respecta a la interactividad, es limitada. Hay posibilidad de difundir los contenidos en Facebook, Twitter y correo electrónico. La participación de los usuarios se produce a través de comentarios y también puede crear una lista de contenidos favoritos.

+tve es la aplicación específicamente interactiva de Radiotelevisión Española disponible para tableta iOS y Android. Es una aplicación corporativa de contenidos con una oferta general dividida en: «Antes», «Ahora» y «Después». El hecho de potenciar que los usuarios compartan contenidos justifica un desplegable situado en la parte inferior de la pantalla que recoge los últimos momentos compartidos, los más populares y el registro de la actividad del usuario.

El contenido que se ofrece en videoteca es limitado, al igual que los contenidos extra, que se limitan a información complementaria relacionada, fotogalerías o making of. Se agrupan en una columna denominada «¿Sabías qué?».

Dadas las características y el objetivo de la aplicación, la interactividad tiene distintas posibilidades. Hay conexión a Facebook, Twitter y Google+. Se pueden compartir fragmentos de los contenidos e interactuar a través de la aplicación con comentarios sobre las capturas. Esos comentarios puede considerarse que dan origen a una comunidad de seguidores.

24 Horas RTVE es una aplicación centrada únicamente en la información. Se estructuran en cinco grandes apartados: «Noticias», donde se recoge lo más destacado del día, «+24 Directos», con emisiones en directo, «TD A la Carta» para seleccionar informaciones, «Mis Noticias», constituido a partir de las preferencias de información marcadas por el usuario, y una pestaña para temas monográficos que en el periodo de observación era el 1-O en Catalunya.

En un menú desplegable lateral también se puede acceder a Informe Semanal, otros programas e información del tiempo y del tráfico.

Los contenidos se pueden compartir a través de Facebook, Twitter y el correo electrónico.

\section{Atresmedia}

Atresmedia cuenta con dos aplicaciones, Atresplayer y Atresmedia conecta. Las dos son apps Corporativas; cuentan con contenidos y opciones interactivas para varios canales del grupo. La finalidad de Atresplayer es contenidos, mientras que para Atresmedia conecta es interactividad. Analizando el 
contenido de la aplicación Atresplayer, cuenta con las siguientes secciones: «Entra», «Directos» (radio y televisión), «Buscador A-Z» y a un menú con diferentes categorías. Además, hay acceso directo a varios programas destacados. Hablando de la estructura del entretenimiento, «Series», «Programas», «Deportes», «Telenovelas», «Sketches», «Cine»y «Más contenido»son las formas de clasificación que aparecen, además de «Orden alfabético».

En cuanto a la publicidad presente, aparece en forma de vídeo al principio y durante la reproducción. Se ofrece servicio a la carta con amplia variedad y cantidad de contenido, en donde el usuario puede acceder a capítulos y programas. Hay que destacar que estos son de producción propia y producción ajena. Al entrar en la sección de algún programa o serie, el usuario tiene a su disposición contenidos extra, concretamente información complementaria como sinopsis y otros extras como montajes especiales, entrevistas, making of, ruedas de prensa o encuentros virtuales. En la app hay disponible la emisión en directo de televisión y radio. En el caso de la televisión, se ofrece la señal de los canales Antena 3, Atreseries, La Sexta, Mega, Nova y Neox, pero no del 100\% por falta de derechos. Además, en algún momento, se ofrecen imágenes en directo de actos noticiosos. En el caso de la radio, están disponibles Onda Cero y Europa FM.

En lo que se refiere a la interactividad, el usuario no puede enviar contenidos como fotografías o vídeos. Sí que tiene opción a compartir en Facebook y Twitter. Además, cuenta con acceso a comunidad virtual. Al estar registrado, el usuario puede comprar contenidos como preestrenos de series y verlos desde la web o la propia app Atresplayer.

Profundizando en la aplicación Atresmedia conecta, está destinada a interactuar con programas o series durante la emisión. Es corporativa, ya que ofrece la posibilidad de interactuar con los contenidos de diferentes canales del grupo. Cuando el usuario entra en la app, encuentra en la página principal la opción «Programa interactivo», siempre y cuando se esté emitiendo un contenido de esas características en ese momento, si no aparecerá la opción «Próximas emisiones». Al participar en esta $a p p$, se pueden ver contenidos extras, así como participar en encuestas, concursos o rankings interactivos. Además, hay opciones de segunda pantalla, ya que se puede interactuar en tiempo real en Twitter y Facebook. También, a través de estas dos redes sociales, se permite compartir cierto contenido de la app.

Estas son las funciones para las que fue creada esta aplicación, pero hay que destacar que en las fechas de análisis, Atresmedia no la utilizó en ningún contenido.

\section{MEdiASET}

Mediaset España dispone de la aplicación Mitele para iOS y Android y, en ambos casos, es exactamente igual en cuanto a su diseño, contenido e interactividad. Esta aplicación tiene carácter corporativo porque concentra su oferta en los contenidos de los programas que se emiten en Telecinco y Cuatro. Sin embargo, no emite programas de otros canales del grupo como FDF, Boing, Energy o Divinity.

Las secciones con las que se encuentra el usuario en la página principal son: «Portada», «En directo», «Mitele Teens», «Programas», «Series», «TV Movies», «Miniseries», «Cine», «Informativos», «Deportes», «Momentazos», «Documentales», «12 meses» y «Ayuda».

La publicidad ${ }^{1}$ aparece en formato video al principio de cada reproducción y al final de la misma. Según el tipo de contenido pueden aparecer dos o tres videos publicitarios antes del programa. Se

${ }^{1}$ Mediaset tiene una plataforma ADLIVE de contenidos publicitarios online. http://www.mitele.es/blog/2012/12/adlivepublicidad-y-contenidos-solo-online/ 
observa que, durante el periodo de emisión de programas con gran audiencia, aparece un anuncio a pantalla completa durante diez segundos. El formato habitual que se ha visto durante el periodo analizado es intersitial, es decir, es un espacio de publicidad a pantalla completa con un periodo de duración muy corto y que su duración variará dependiendo del tiempo de carga del usuario.

Se ofrece un servicio a la carta con gran variedad de contenido. Mediaset tiene los derechos de numerosas obras de ficción emitidas durante los últimos años. Destacar Hospital central (20 temporadas), Al salir de clase (1199 capítulos). Además, cuenta también con series de producción más reciente como El accidente. Tanto en la sección «Ficción» como «Entretenimiento» se pueden ver programas de producción propia y producción ajena de los que se ha hecho una versión para Mediaset, como. por ejemplo, El método Osmin, El líder de la manada, etc.

$\mathrm{Al}$ entrar en cualquier contenido es posible acceder al visionado de los capítulos en una de las pestañas. Siempre está disponible la posibilidad de acceder a información complementaria en el icono «tinfo», donde se puede encontrar la sinopsis, duración, edad recomendada y periodo de disponibilidad.

En esta $a p p$, en general, no hay contenidos extra salvo en alguna serie como Dreamland que tiene un making of del primer capítulo; El chiringuito de Pepe oferta también un «Como se hizo» y Aida «Mejores momentos de Dani Martínez». No es una oferta habitual. En la emisión en directo se aprecian los contenidos de cada uno de los canales que aparecen con esta posibilidad de conexión: Telecinco, Cuatro, GH VIP, Premier Casino, Fútbol mitele y 8tv. La emisión no es permanente y se publicitan horas determinadas para la misma. Algunos de estos canales no tienen emisión en directo, sino un bucle de contenido.

Analizando la interactividad de esta aplicación se observa que no permite compartir enlaces ni contenidos en Facebook y Twitter. Sí se puede hacer a través de la página web de Mediaset, que es hacia donde redirige la aplicación cuando se quiere compartir contenidos. La participación de los usuarios es a través de comentarios y es necesario estar registrado en la aplicación Mitele.

En esta $a p p$ no figura acceso directo a contenidos de pago y alquiler. Este puede realizarse a través de la web (www.mitele.es) y de este modo es posible verlo en la aplicación.

Desde el año 2013 Mediaset apostó por generar interactividad con los espectadores y creó la aplicación interactiva Mio TV para que los usuarios pudiesen interactuar con los contenidos del grupo. Se podía descargar en smartphones Android e iOS, así como en tabletas Android. Permitía ARG (Alternate Reality Game), compartir contenido, etc. En el momento de realizar este estudio, los contenidos y posibilidades que mantenía esta app habían desaparecido y en la actualidad se mantiene como una aplicación que permite la compra de merchandising y gestión de envíos.

\section{RTP}

La emisora pública Radio Televisión Portuguesa (RTP) tiene dos aplicaciones para tablet, RTP Notícias y RTP Play, con algunas diferencias de funcionalidades y contenidos que permiten considerar a la segunda como más avanzada en la explotación de contenidos en este tipo de dispositivos móviles.

RTP Notícias es una aplicación corporativa que se centra en la oferta informativa de la cadena. De hecho, las secciones que presenta en el menú general («Directo», «Notícias», «Video», «Audio»y «Desporto») ofrecen noticias en video y audio. Hay una videoteca de noticias extensa, con unos contenidos que pueden ser compartidos en Twitter y Facebook y enviados a través del correo electrónico, por lo que la interactividad es limitada. Hay una opción que permite enviar temas a la cadena por parte de los usuarios. 
RTP Play es la aplicación para tableta avanzada de la radiotelevisión portuguesa. Su propuesta de secciones de la oferta general es más amplia que en la app anterior («Pesquisar», «Inicio», «On demand», «Em Direto», «Programas» e «Info», donde se ofrecen datos de contacto y técnicos de la aplicación). El grueso de la oferta de contenidos se concentra en «Inicio», y «On demand».

A partir de «Inicio» podemos entrar en un submenú dividido en «Gerais», «Informaçao», «Ficçao», «Entretenimento» e «ZigZag» (infantil). Ficçao muestra una limitada selección de series y un programa de humor; «Entretenimento» tiene también una reducida oferta de programas, y en ambos casos se constataron problemas de acceso en los días de observación.

En «On Demand» se establecen las categorías «+Recentes» y «+Populares»; la videoteca es extensa en el conjunto global de todas las secciones, con capítulos de series y programas de producción propia en su gran mayoría, de los cuales se facilita información sobre su contenido. Se ofrece la posibilidad de acceder a las emisiones en directo de radio y televisión; es posible compartir los contenidos en Twitter y enviarlos por correo.

\section{TVI}

El canal TVI cuenta con dos aplicaciones, TVI y TVI player. Las dos son corporativas ya que tienen presencia contenidos de todos los canales del grupo. La finalidad de las dos apps es contenidos. Profundizando en la aplicación TVI, las secciones de la oferta general son tres: «Vídeos», «Guía TV»y «Direto».

No hay disponible un apartado de entretenimiento, ya que el contenido audiovisual aparece en la pantalla principal a modo de galería con el único criterio de la fecha de subida, no cuenta por lo tanto con categorías «Destacadas» o «Más vistos».

En esta aplicación no se aprecia publicidad. El contenido on demand es escaso. Se compone de fragmentos de programas y todo el que se oferta es de producción propia. No dispone de contenidos extra.

Esta aplicación no ofrece emisión de canales en directo a pesar de que cuenta con una opción en el menú denominada «Direto». Cuando el usuario entra en esta sección recibe un aviso de que para ver canales en directo debe visitar la otra aplicación del grupo, TVI Player.

Hablando de la interactividad, el usuario no tiene a su disposición ninguna de las herramientas interactivas analizadas. Si comparamos la aplicación en iOS y Android, no se aprecia ninguna diferencia.

Analizando en detalle TVI player, tienen presencia los canales de televisión de TVI como son: TVI, TVI 24, TVI ficçao y TV Internacional. La finalidad principal es ofrecer contenidos. Las secciones de la oferta general son: «Inicio», «Últimos», «Programas», «Exclusivos» y «Guía TV». No se puede hablar de la estructura del entretenimiento ya que la oferta no se organiza por su tipología, temática, etc. $\mathrm{Al}$ acceder a un programa, en muchos de ellos hay tres pestañas: «Episodios», «Populares»y «Clipes». Otra forma de clasificar el contenido es orden alfabético. Esta se utiliza en el apartado «Programas» donde se clasifican primeramente en los cuatro canales TVI, TVI 24, TVI ficçao, TV Internacional y además, Exclusivos. Dentro de cada uno aparecen clasificados por orden alfabético.

La publicidad aparece en forma de banner. Se puede acceder a multitud de contenidos ya emitidos, todos ellos de producción propia. La gran mayoría ofrecen contenidos extras como información complementaria o fragmentos. Se puede seguir en directo la señal de dos de los cuatro canales de televisión de los que disponen, TVI y TVI 24. En las tabletas con sistema iOS, además se puede seguir también en directo en canal TVI reality. Esta es la única diferencia que se encuentra entre la aplicación en iOS y Android. 
En cuanto a la interactividad, hay que destacar que el usuario para acceder a la app debe estar registrado en la misma. Esto lo puede hacer por correo, Facebook, Google+ o Nos. Una vez que accede puede compartir vídeos y otra información de la aplicación por redes sociales y correo. Además, puede subscribirse a un boletín con las noticias y novedades del canal.

\section{SIC}

El grupo SIC (Sociedad Independiente de Comunicación) gestiona una oferta de canales temáticos, disponibles en los principales operadores de cable y satélite. Tramita diversos canales como SIC Radical, dirigido a un público joven; SIC Mulher con un target femenino; SIC K con temática infantil y el canal informativo SIC Noticias que se creó en el año 2001. También Gestiona SIC Caras una empresa conjunta entre SIC y la revista del corazón Caras.

SIC Noticias es la aplicación para tablets de este grupo. Es una app de canal, no corporativa y sus contenidos son informativos. Las secciones que muestra en el menú general son: «Homepage», «Últimas», «Opiniao», «Programas»y «Prime».

En las tres primeras secciones se pueden ver diferentes noticias del día. En concreto «Homepage» ofrece información escrita e imágenes de cada una de las noticias de un modo similar a como se concibe en prensa escrita. Cuando se visiona la noticia seleccionada surge en la margen derecha de la pantalla varias noticias con una temática similar. En concreto, se subdivide en «Últimas noticias», «Destaques», «Programas», «Contas popança», «Ediçao da manhá», «Reportagem especial», etc. que brinda piezas de video por temáticas: «Cultural», «Mundo», «Economía», «País», etc.

Noticias mantiene un menú («Cultural», «Deportes», «Prime», «Economía», «Mundo», «País») muy similar a las secciones de los periódicos. Tiene una videoteca extensa. En esta sección se pueden ver las noticias a pantalla completa o estructurada en galería.

La aplicación está asociada a SAPO que es un buscador portugués proveedor de servicios y productos para Internet que es el más grande de Portugal. En cuanto se accede a la app hay un indicador con el logo que así lo indica.

Además de «Noticias» encontramos la sección «Programas», en la que se observan cerca de treinta títulos diferentes con programas de un marcado contenido informativo en el que se publicita el título del contenido. Los programas temáticos versan sobre economía, salud, entrevistas, deporte, magacín y moda. Permite realizar la búsqueda del programa que desees. Cuando se accede a cada uno de estos contenidos se abre una nueva ventana que ofrece información sobre el programa y en la parte inferior se ofrecen ediciones anteriores con un titular que hace referencia al contenido de cada uno de los capítulos.

La videoteca no es extensa y es necesario esperar a que se carguen los programas a medida que se busca información sobre un programa ya emitido. Se comprueba que la oferta es igual en iOS y Android.

Todos los contenidos pueden ser compartidos en redes sociales (Facebook, Google+, Twitter, Linkedin, Pinterest, Instagram) y ser enviados a través del correo electrónico. No existe la posibilidad de interactuar con el programa desde la aplicación.

Desde la pestaña «Guía Tv» se puede acceder a la programación de su canal y tiene a disposición una estructura limitada porque ofrece la programación del día anterior a la búsqueda y 5 días posteriores. 


\section{ConCLUSIONES}

El análisis de las aplicaciones propuestas confirma que los contenidos que difunden las operadoras fueron emitidos anteriormente en la programación convencional. En términos generales, no hay un formato propio para estos dispositivos, más allá de la segmentación y fragmentación de los programas.

También se concluye que existe una homogeneidad de contenidos en las aplicaciones, aunque el sistema operativo sea distinto. Es decir, las aplicaciones de RTVE, Atresmedia, Mediaset, RTP, TVI y SIC son semejantes en su oferta para los sistemas operativos iOS y Android, de tal manera que no se hace uso de diferentes interfaces o contenidos. Es coherente con el proceso de producción y evita diferenciar las propuestas por dispositivos, con lo que ello supondría para los usuarios. Sin embargo, sí hay diferentes finalidades en las aplicaciones. Los principales operadores en el ámbito público y comercial, RTVE y Atresmedia, ofrecen apps distintas según el objetivo primordial que persigan: difusión de contenidos únicamente o difusión de contenidos e interactividad. Rtve.es | Tableta y Atresplayer están destinadas fundamentalmente a mostrar contenido, mientras que +tve y Atresmedia conecta son propuestas interactivas. En el caso de las televisiones portuguesas, a excepción de RTP, TVI y SIC noticias, centran sus aplicaciones en la oferta de contenidos. En los operadores públicos español y portugués se ofrece una aplicación exclusivamente dedicada a noticias e informativos.

La decisión empresarial de RTVE, Atresmedia y RTP está relacionada con la progresiva potenciación del papel del usuario/espectador dentro del consumo de contenidos audiovisuales y con el aprovechamiento de las posibilidades de los dispositivos móviles como plataformas de recepción. Por lo que respecta a RTVE, la corporación pública ha actuado como verdadero motor del desarrollo de la televisión en internet y en distintos dispositivos, cumpliendo con una de las tareas que le corresponde como medio de comunicación público.

En el caso de + tve, el usuario puede compartir fragmentos de los programas o series emitidas. Del análisis comparativo de la oferta de entretenimiento y herramientas interactivas de las apps móviles para tableta analizadas, se colige que RTVE ha desarrollado una oferta global de contenidos e interactividad más avanzada.

Llama la atención que un grupo audiovisual tan potente en España como Mediaset vaya por detrás en este aspecto, ya que su aplicación, Mitele, está orientada solo hacia los contenidos. En este caso hay que señalar que Mediaset intentó desarrollar una aplicación interactiva para dispositivos móviles denominada Mio TV pero por circunstancias adversas y falta de confianza en los resultados económicos se paralizó su desarrollo.

Aunque la interactividad se potencia en la aplicación que hemos señalado en el párrafo anterior, las apps dedicadas a contenidos también tienen posibilidades interactivas, ya que todas ellas permiten compartir los contenidos, noticias, etc., por medio de redes sociales (Facebook y Twitter). Asimismo, en el caso de Rtve.es | Tableta, también por correo electrónico. Destacar que Atresplayer y TVI disponen además de comunidad virtual. La primera de ellas permite visualizar los contenidos adquiridos mientras que en TVI es obligatorio el registro para acceder a la app.

El carácter corporativo prima en la presentación de la oferta audiovisual en las tabletas ya que todas las aplicaciones objeto de estudio tienen esta orientación de grupo; ofrecen contenidos de los diferentes canales que pertenecen a una misma operadora de televisión. En este sentido se constata que la oferta que pone en liza TVE es de mayor amplitud y calado que las emisoras comerciales. Con todo, el análisis desarrollado revela que hay una serie de categorías presentes en todos los emisores considerados: series, programas y documentales. 
Como corresponde a emisoras comerciales, tanto en el caso de Atresplayer como en el de Mitele de Mediaset, se destacan ofertas que reúnen altos porcentajes de audiencia como telenovelas o deportes. Hay que significar la apuesta por contenidos emergentes y propios del audiovisual en internet, como el espacio para webseries de Atresplayer o LVP (Liga de Videojuego Profesional) de Mediaset que en este caso se dirige a un target joven muy específico.

La difusión de contenidos a través de tabletas, smartphones o mediante la televisión conectada permite poner a disposición del consumidor contenidos extra como información complementaria. Es lo que acontece con Rtve.es | Tableta, Atresplayer y Mediaset. En el caso concreto de Rtve.es | Tableta, permite acceder a fragmentos de programas; Atresplayer pone a disposición del usuario montajes extras como making of o ruedas de prensa.

La publicidad tiene distinto valor en las propuestas de los canales considerados. Hay un uso de los mensajes publicitarios en las emisoras comerciales y está ausente en el operador público RTVE. La percepción de la publicidad por parte del espectador es menos invasiva que en la emisión convencional; no hay grandes bloques de anuncios, estos se ofrecen al inicio de los programas y existe la posibilidad de entrar en la página web del anunciante, con lo que se abre incluso la opción de adquirir productos de la marca. Estamos ante una publicidad basada en la capacidad de generar empatía e interés con el espectador, no en la saturación de spots en un espacio de tiempo limitado propia de la emisión lineal y la posición pasiva del espectador.

Por lo que respecta a la observación de las aplicaciones para tabletas de las emisoras portuguesas cabe concluir que no existen prácticamente diferencias en los contenidos, interfaces y capacidades de interacción entre los sistemas iOS y Android. Las singularidades se focalizan en los canales disponibles en la emisión en directo de la aplicación TVI Player, ya que en iOS hay acceso a tres canales mientras que en Android a dos.

Al igual que ocurre en el caso español, la emisora pública, la RTP, ofrece una doble oferta: una aplicación corporativa centrada en contenidos, aunque en este caso únicamente informativos, y otra eminentemente interactiva; sin embargo, el desarrollo y las capacidades están por debajo de las aplicaciones de RTVE.

El uso de las redes sociales como cauce de participación de la audiencia y difusión de contenidos está menos desarrollado en las emisoras portuguesas que en las españolas. En el caso luso, son los canales comerciales quienes más explotan esta vertiente de la nueva televisión; el grupo SIC permite que los programas y las noticias se compartan en Facebook, Twitter y correo electrónico, pero no existe comunidad virtual ni la posibilidad de interactuar con los contenidos. Por lo que respecta a TVI su interactividad está concentrada en TVI Player, en la que se puede compartir contenido de correo electrónico o redes sociales y existe comunidad virtual.

Los contenidos a disposición de los usuarios son más amplios en RTP que en el conjunto de las emisoras comerciales. En el caso de TVI las secciones de la oferta general son escasas y en las dos apps no podemos hablar de secciones de entretenimiento. La oferta a la carta está más desarrollada, principalmente en RTP Play y TVI Player. En ambas emisoras existe la posibilidad de acceder a los directos.

SIC presenta una aplicación muy enfocada a la información donde es posible ver en directo la programación o acceder a los contenidos de ficción, entretenimiento y concursos. Cuenta con varias aplicaciones «SIC Noticias», «Sic Mulher», «Sic Caras» en las que ofrece contenidos específicos a los usuarios por contenido y target.

El análisis de las aplicaciones para tabletas de las emisoras portuguesas nos permite concluir que su oferta de contenidos e interactividad es menor que en el caso de las españolas, hay menos conteni- 
dos a disposición de los usuarios y la utilización de las redes sociales está orientada solo a compartir, sin la capacidad de establecer una conexión simultánea e inmediata. Es significativo que en uno de los casos considerados no exista posibilidad real de acceder al directo de las emisiones. Las apps de las televisiones portuguesas atraviesan un estado de evolución integral menos avanzado; es necesario destacar que en ambos países las televisiones públicas tienen un papel relevante en la potenciación de los contenidos en movilidad y que una de las dos cadenas comerciales sobresale sobre la otra en la oferta, Atresmedia en el caso español y TVI en Portugal.

\section{BibliografíA}

\#IAB Estudio Mobile. (2015). International Advertising Bureau y Elogia Marketing 4 Commerce. Aguado, J. M. y Martínez, I. (2008). La cuarta pantalla: industrias culturales y contenido móvil. En J. M. Aguado e I. J. Martínez (coords.), Sociedad móvil: Tecnología, identidad y cultura (pp. 187-220). Madrid: Biblioteca Nueva.

Barbosa, S. y Seixas, L. (2013). Jornalismo e dispositivos móveis: percepções, usos e tendencias. En S. Barbosa y L. Mielniczuk (orgs.), Jornalismo e Tecnologias Móveis (pp. 51-74). Covilhã: Livros LabCom.

Canavilhas, J. (2009). Contenidos informativos para móviles: estudio de aplicaciones para iPhone. Revista Textual \& Visual Media, 2, pp. 61-80. Consulta 13 de febrero de 2016. http://hdl.handle.net/10400.6/684

Canavilhas, J. y Santana, D. C. (2011). Jornalismo para plataformas móveis de 2008 a 2011: da autonomia à emancipação. Revista Líbero, 14(28).

Canavilhas, J. (2012). Jornalismo para dispositivos móveis: informação hipermultimediática e personalizada. Actas do IV Congreso Internacional Latina de Comunicación Social. Consulta 11 de enero de 2016. http://www.revistalatinacs.org/12SLCS/2012 actas.html

Cebrián, M. y Flores, J. M. (2011). Periodismo en la telefonía móvil. Madrid: Editorial Fragua.

Costa-Sánchez, C. (2013). Prensa en el Smartphone. Modelos aplicados en los diarios españoles y necesidades de desarrollo. Icono 14, 11(2). pp. 07-30. Consulta 11 de febrero de 2016. doi: https://doi.org/10.7195/ri14.v11i2.591

Costa-Sánchez, C. (2014). Las singularidades del medio móvil: Integración multimedia, personalización, geolocalización y participación. Estudio de su presencia en las apps de la prensa española. Palabra Clave, 17(3), pp. 672-694. doi: https://doi.org/10.5294/pacla.2014.17.3.5

Costa-Sánchez, C., Rodríguez-Vázquez, A. I. y López-García, X. (2016). Dispositivos móviles: el nuevo reto para la industria de la prensa y del libro en España. Palabra Clave, 19(2), pp. 526-555. doi: https://doi.org/10.5294/pacla.2016.19.2.8

Costa-Sánchez, C., Díaz-González, M. J. y Videla-Rodríguez, J. J. (2012). La investigación sobre dispositivos móviles desde las ciencias de la comunicación: aportaciones realizadas y retos futuros. En III Congreso Internacional de la Asociación Española de Investigación de la Comunicación. Tarragona: AEIC.

Cuello, J. y Vittone, J. (2013). Diseñando apps para móviles. Autoedición.

Fernández-Planells, A. y Figueras Maz, M. (2014). De la guerra entre pantallas a la sinergia entre pantallas. El multitasking en jóvenes. En A. Huertas Bailén y M. Figueras Maz (eds.), Audiencias juveniles y cultura digital (pp. 87-105). Institut de la Comunicació, Universitat Autònoma de Barcelona.

Fidalgo, A. (2009). O celular de Heidegger - comunicaçao ubíqua e distância existencial. Matrizes, 3. pp. 81-98. doi: http://dx.doi.org/10.11606/issn.1982-8160.v3i1p81-98 
Fidalgo, A. y Canavilhas, J. (2009). Todos os jornais no bolso: pensando o jornalismo na era do celular. Jornalismo on-line: modos de fazer, pp. 96-146.

Ford, R. y Wiedemann, J. (2011). Apps para dispositivos móviles: casos de estudio. Köln: Taschen Gmbh.

Formoso, F. (2015). Los contenidos transmedia en la ficción de los canales generalistas en España. adComunica. Revista Cientifica de Estrategias, Tendencias e Innovación en Comunicación, 10, pp. 41-59. doi: http://dx.doi.org/10.6035/2174-0992.2015.10.4

Futuro digital. España 2015. (2015). comScore. Consultado 19.04.2016 en https://www.comscore.com/esl/Prensa-y-Eventos/Blog/Futuro-Digital-Europa-2015

García-Torre, M. (2014). Análisis de las posibilidades de interacción del usuario en los portales web y aplicaciones móviles de la Compañía Radio Televisión de Galicia y de Euskal Irrati Telebista. En J. Rodríguez (coord.), Creaciones audiovisuales actuales. Madrid: Editorial ACCI.

Igarza, R. (2009). Burbujas del ocio. Buenos Aires: La Crujía Ediciones.

Loosen, W. y Schmidt, J. (2012). (Re-) Discovering the audience. The relationship between journalism and audience in networked digital media. En O. Westlund (ed.), Information, Communication \&o Society, 15(6), pp. 867-887.

Márquez, I. (2015). Una genealogía de la pantalla. Del cine al teléfono móvil. Barcelona: Editorial Anagrama.

Nel, F. y Westlund, O. (2013). Managing New(s) Conversations: The Role of Social Media in News Provision and Participation. En Friedrichsen, M. y W. Mühl-Benninghaus (ed.), Handbook of social media management. Springer.

Pérez-Latre, F. J. y Sánchez Tabernero, A. (2016). The Business of Media Final. Change and Challenges. Porto: Editorial Board.

Piñeiro-Otero, T. (2015a). De la radio convencional a la móvil. Usabilidad, multimedialidad y distribución de contenidos en las radioapps portuguesas. Observatorio (OBS*), 9(3), pp. 47-66. doi: http://dx.doi.org/10.7458/obs932015836

Piñeiro-Otero, T. (2015b). Información, multimedialidad y personalización en las radioapps de España y Portugal. El Profesional de la Información, 24(4).

Salaverría, R. (2016). Los medios de comunicación que vienen. En Ch. Sádaba Chalezquer, J. A. García Avilés y M. P. Martínez Costa (coords.), Innovación y desarrollo de los cibermedios en España. Pamplona: Eunsa.

Salaverría, R. y Sádaba, Ch. (2016). La innovación y los cibermedios. En Ch. Sádaba Chalezquer, J. A. García Avilés y M. P. Martínez Costa (coords.), Innovación y desarrollo de los cibermedios en España. Pamplona: Eunsa.

Silva Rodríguez, A., López García, X., Westlund, O. y Ulloa Erazo, N. G. (2016). Iniciativas en la comunicación móvil: mapeo de los canales, el contenido, la conversación y el comercio en Europa y los Estados Unidos. Observatorio (OBS*), 10(1), pp. 201-217.

Televidente 2.0. Audiovisual consumption trends in Spain ( $8^{\circ}$ wave). (2015). The Cocktail Anlysis. Consulta el 10.04.2016 en http://tcanalysis.com/blog/posts/televidente-2-0-audiovisualconsumption-trends-in-spain

TV or not TV, that's the question. (2015). Zenith. Consultado el 16.04.2016 en https://blogginzenith.zenithmedia.es/tv-or-not-tv-thats-the-question-claves-de-una-nuevatelevision/

Vacas, F. (2007). Teléfonos móviles: la nueva ventana para la comunicación integral. Madrid: Agapea.

Videla-Rodríguez, J. J. \& Piñeiro-Otero, T. (2013). Apps de radio. Movilidad para un medio radiofónico sin límites. PRISMA.COM, 17. 
Videla-Rodríguez, J. J., García-Torre, M. y Formoso, M. J. (2014). Contenidos informativos en movilidad de las televisiones públicas. En J. Rodríguez Terceño y A. Fernández Paradas (coords.), Nuevas culturas y sus nuevas lecturas (pp. 695-706). Madrid: Editorial McGraw-Hill.

Vilas Fumas, P.; Fuentes Cortina, G. (2013). La convergencia en los nuevos soportes y en las redes sociales. Cómo la tecnología facilita la convergencia. En J. M. Legorburu Hortelano (coord.), Modelos de convergencia de medios en España. Digitalización, concentración y nuevos soportes. Madrid: CEU Ediciones.

Westlund, O. (2011). Cross-media news work-Sensemaking of the mobile Media (R)evolution. Tesis Doctoral. Gothenburg: University of Gothenburg.

Westlund, O. (2015) News consumption in an age of mobile media: Patterns, people, place, and participation. Mobile Media \& Communication, 3, pp. 151-159. doi:

https://doi.org/10.1177/2050157914563369 